\title{
LA-UR-18-22730
}

Approved for public release; distribution is unlimited.

Title: $\quad$ Safety Applications Project Performance Benchmark

Author(s): $\quad$ Smith, Brandon Michael

Intended for: $\quad$ Report

Issued: 
Disclaimer:

Los Alamos National Laboratory, an affirmative action/equal opportunity employer, is operated by the Los Alamos National Security, LLC for the National Nuclear Security Administration of the U.S. Department of Energy under contract DE-AC52-06NA25396. By approving this article, the publisher recognizes that the U.S. Government retains nonexclusive, royalty-free license to publish or reproduce the published form of this contribution, or to allow others to do so, for U.S. Government purposes. Los Alamos National Laboratory requests that the publisher identify this article as work performed under the auspices of the U.S. Department of Energy. Los Alamos National Laboratory strongly supports academic freedom and a researcher's right to publish; as an institution, however, the Laboratory does not endorse the viewpoint of a publication or guarantee its technical correctness. 


\section{Safety Applications Project Performance Benchmark}

Pagosa Shaped Charge

Brandon Smith

March 22, 2018 


\section{The performance benchmark was conducted using the shaped charge Pagosa sample problem.}

- This problem was chosen because it is a wellcharacterized simulation with a zone count ideal for strong scaling studies.

- Geometry is axisymmetric but simulation is run in 3D.

- Simulation runs to $30 \mu \mathrm{s}$ with EnSight output every $1 \mu \mathrm{s}$.

- Materials are copper liner, composition B charge, tetryl booster, stainless steel case, and void.

- The spatial domain of the $1 \mathrm{~mm}^{3}$ mesh has been increased for scaling purposes from $63 \times 62 \times 248$ to $64 \times 64 \times 256$ zones.

- $1.05 \mathrm{M}$ zones

- Previous Work:

Kelsey, Robert. "Performance Analysis of the Pagosa Application." SIAM Conference on Parallel Processing for Scientific Computing. February 18, 2014. LA-UR-14-20808.
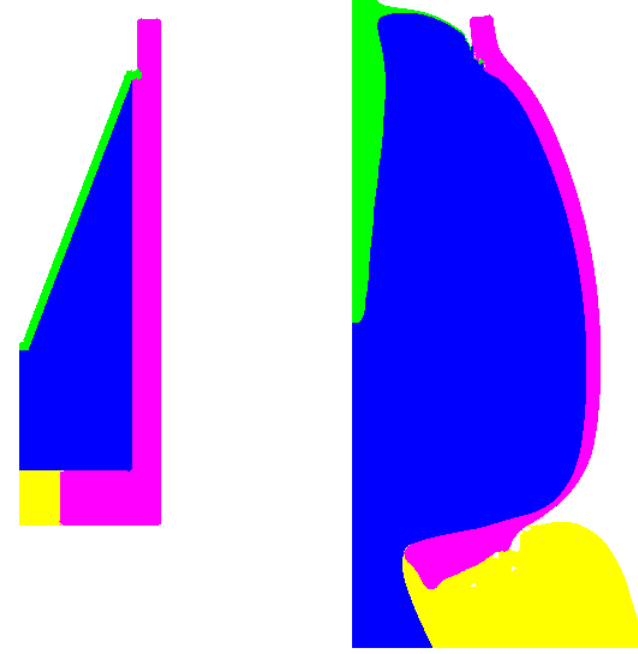


\section{Pagosa strong scaling was examined using the shaped charge sample problem.}

- This exercise used Pagosa 17.3.2 releases for all executables (February 14, 2018).

- A typical Pagosa simulation includes three executables.

- Gen: Determine point-sampled volume fractions and programmed burntimes.

- Pagosa: Integrate hydrodynamic equations through time.

- GD_ES: Convert graphics dump to EnSight output.

- Pagosa simulations widely vary depending on the application.

- Zones: $100-1.5 \mathrm{~B}$

- MPI Ranks: 16 - 500k

- Runtime: 10 seconds - 1 month

- Zones/Rank: 256 - 64k

- Three machine configurations were compared in this study.

- Snow is CTS-1 architecture with 36 cores per node, 1 thread per core.

- Trinitite HW is ATS-1 architecture with 32 codes per node, up to 2 threads per core.

- Trinitite KNL is ATS-1 architecture with 68 cores per node, up to 4 threads per core.

- These data were obtained when Snow was utilized to capacity, but Trinitite's queue was nearly empty. 


\section{Pagosa strong scales well down to 4k zones/rank on} Snow.
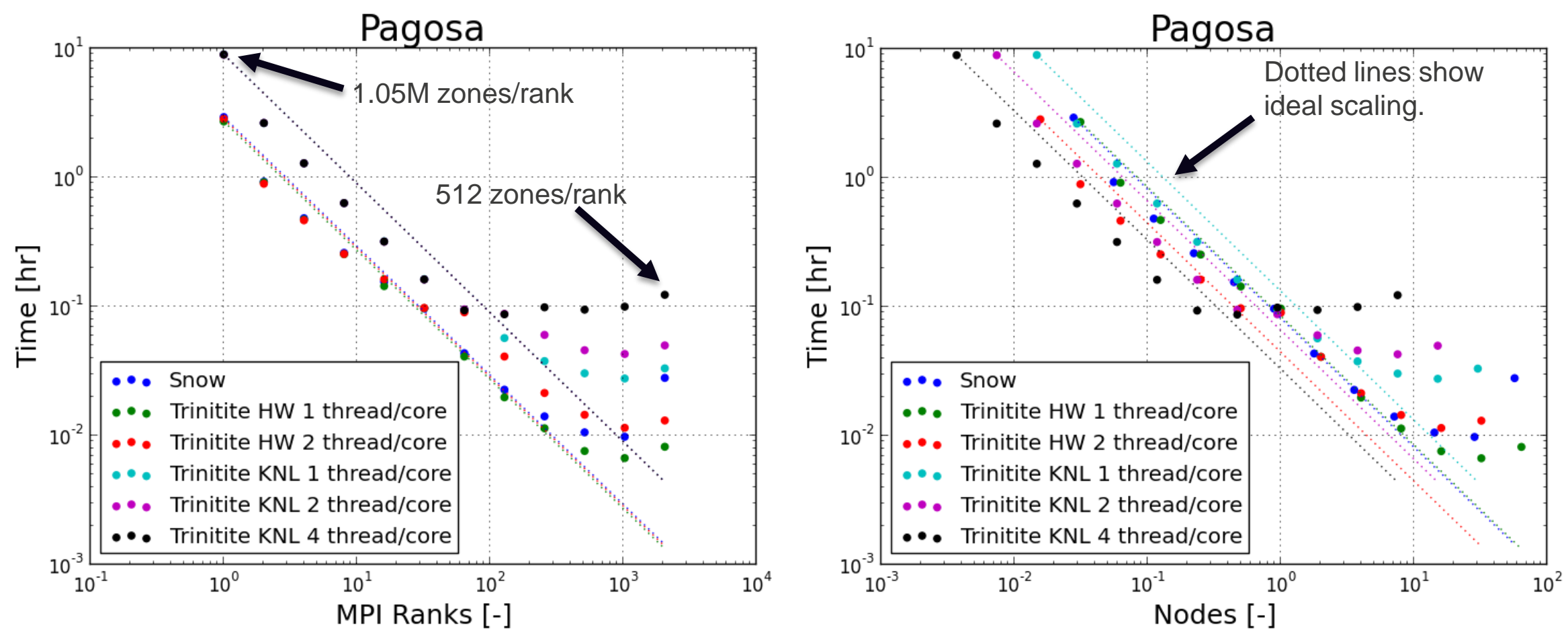

- Threading does not appear to be advantageous for HW or KNL nodes.

- On a per-node basis, the ratio of KNL/HW runtime varies from unity (1 node) to $\sim 4.5$ ( $\sim 32$ nodes). 


\section{If file output is excluded, Pagosa strong scales well through 512 zones/rank.}
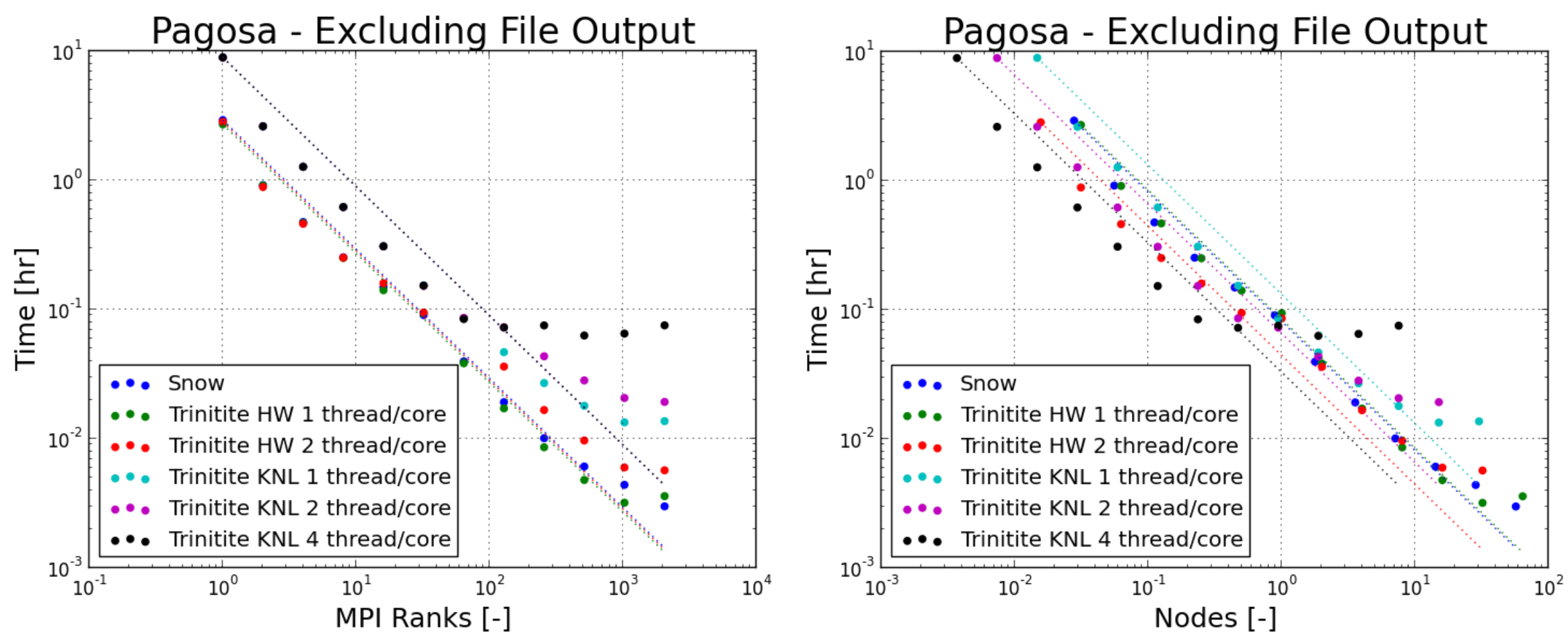

- These data omit the time to write graphics dump files.

- Graphics dump files are post-processed by GD_ES into EnSight output.

- At low zones/rank, ideal scaling is disrupted due to file IO and communication. 


\section{Gen does not strong scale well but does not represent a large portion of overall Gen/Pagosa/GD_ES time.}
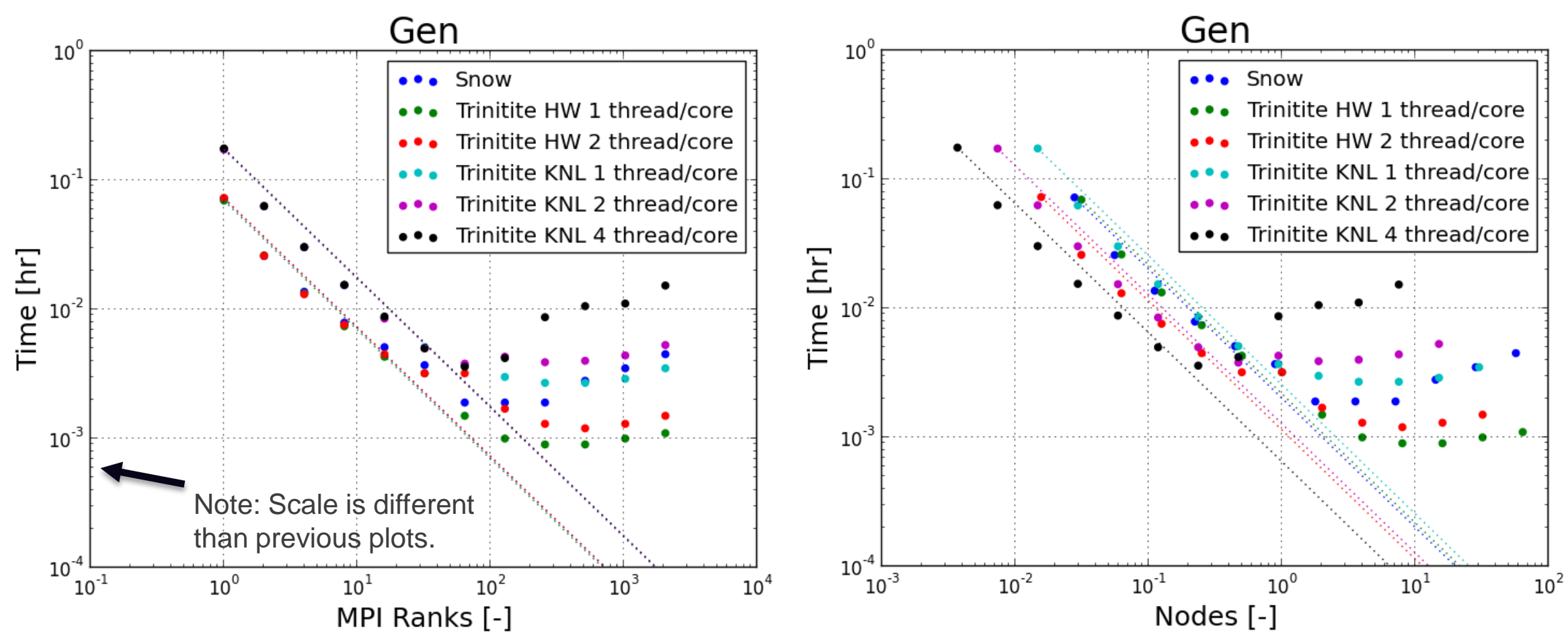

- Bringing Gen and GD_ES into the Pagosa executable would avoid significant file IO and improve the user experience. 


\section{GD_ES does not strong scale well and requires significant execution time.}
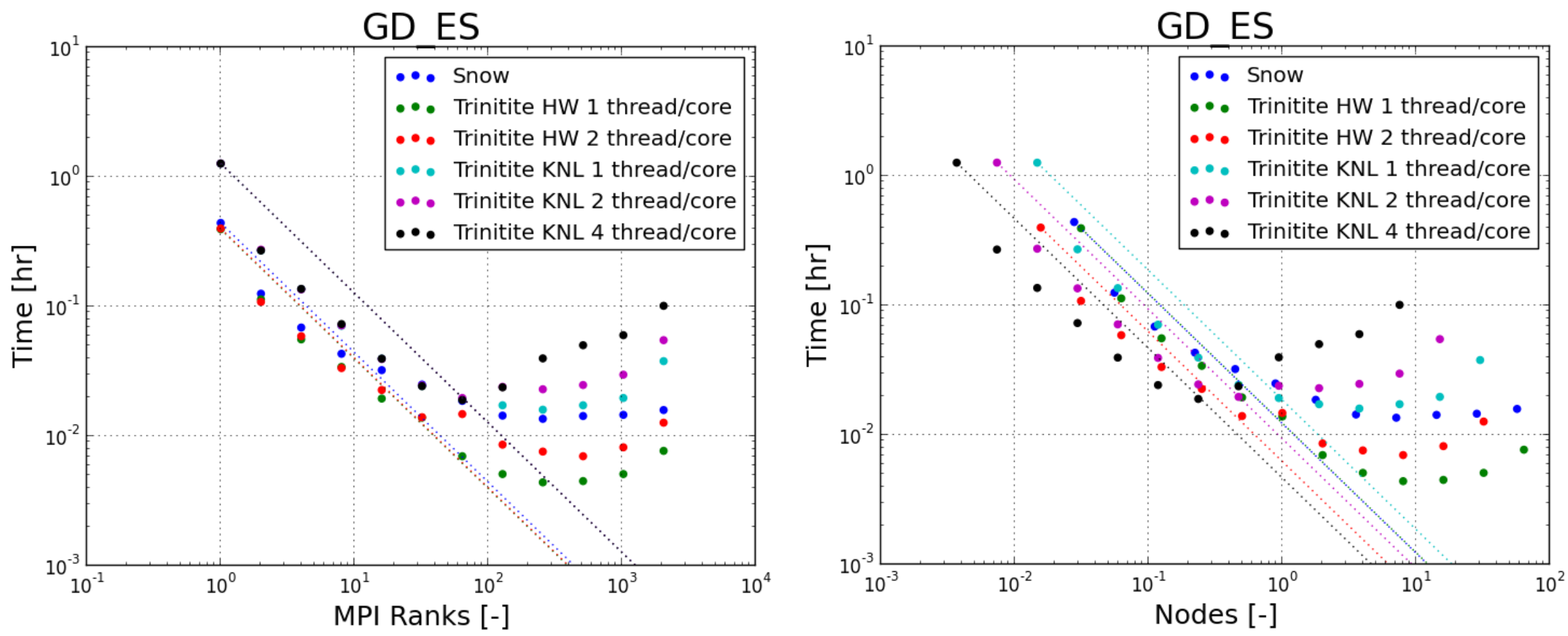

- GD_ES reads graphics dumps in parallel but writes EnSight files on rank 0.

- At 512 ranks and greater on Snow, converting graphics dumps to EnSight output requires more execution time than Pagosa itself. 


\section{Pagosa performance is sensitive to spatial decomposition.}
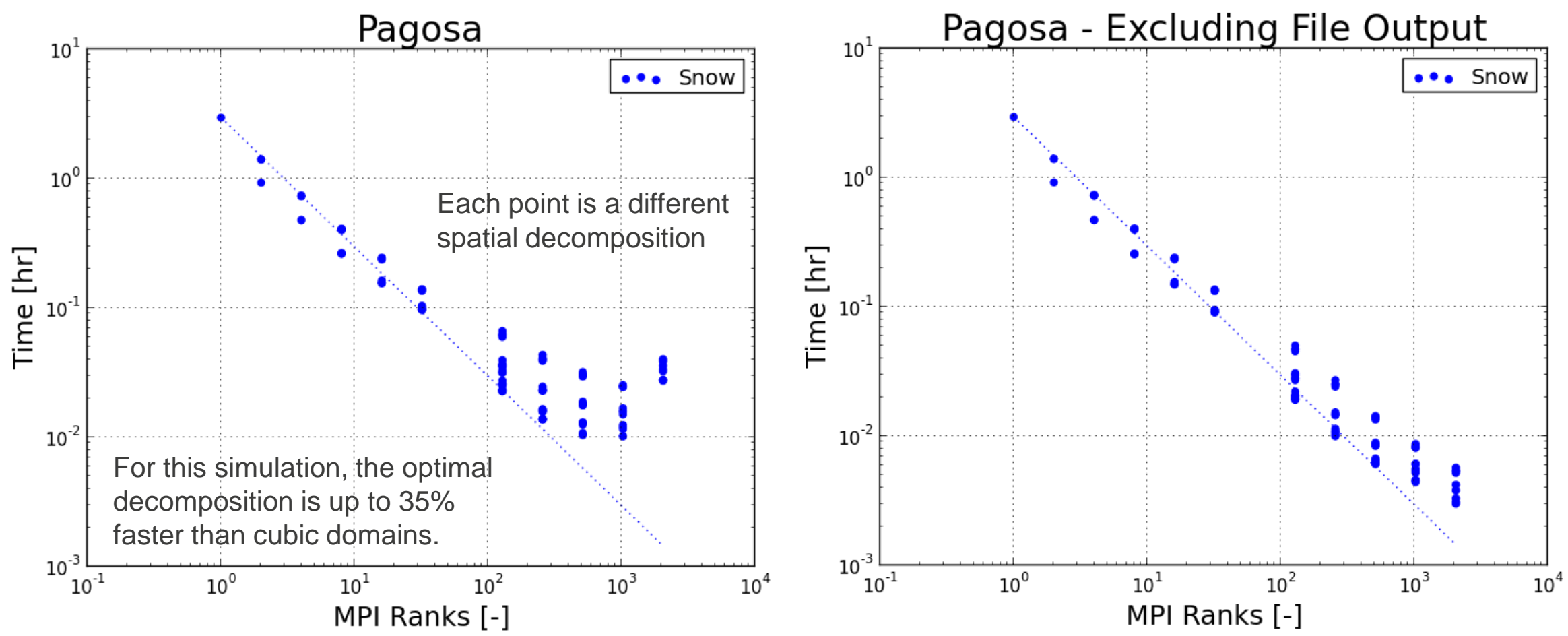

- In all cases, the optimal decomposition has two ranks in the x-direction.

- This observation refutes the lore that cubic spatial domains are most efficient.

- Excluding this slide, all plots use the optimal spatial decomposition. 


\section{Several options were explored on the Trinitite KNL partition.}
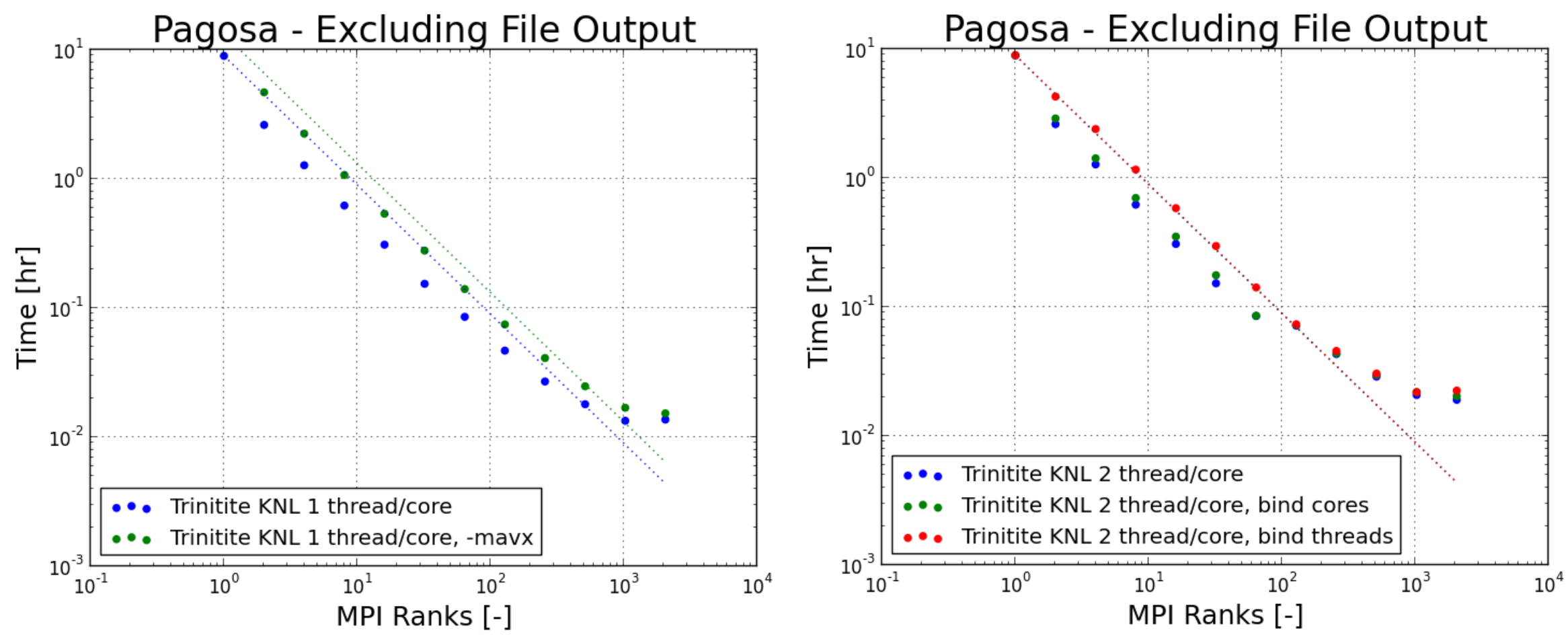

- The -mavx Intel compiler flag did not improve performance.

- Explicit task affinity options --cpu_bind=cores and --cpu_bind=threads did not improve performance. 


\section{What have we learned?}

- Excluding file output, Pagosa strong scales well down to 512 zones/rank.

- Threading does not appear advantageous for HW or KNL nodes.

- On a per-node basis, the ratio of $\mathrm{KNL} / \mathrm{HW}$ runtime varies from unity (1 node) to $\sim 4.5$ ( $\sim 32$ nodes).

- Gen scales poorly but represents a small portion of overall execution time.

- GD_ES scales poorly and represents a large portion of overall execution time. - Bringing Gen and GD_ES inline would avoid significant IO and improve the user experience.

- Pagosa performance depends on the spatial decomposition, likely due to cache size vs. array size considerations. In this study cubic domains were not optimal.

- Compiling with -mavx did not improve performance on Trinitite KNLs. - This may not be surprising-KNL uses AVX512 natively, not AVX or AVX2.

- --cpu_bind=cores and --cpu_bind=threads did not improve performance on Trinitite KNLs. 